öGUM-Mitteilungen

Österreichische Gesellschaft für Ultraschall

\title{
in der Medizin
}

\section{Sonografie-Kurse nach den Richtlinien der ÖGUM}

detaillierte Infos im Kurs-Kalender unter: www.oegum.at/ausbildung/kurskalender. html

\section{Abdomen}

27.-28.09.2019 Salzburg: ÖGUM-Aufbaukurs Abdomen

Leitung \& Auskunft: OA Dr. Hollerweger, kontakt@bhb-sonokurse.at
26.-28.09.2019 Hohenems: Abdomensonografie-Grundkurs

Leitung \& Auskunft: OA Dr. Gehmacher, sekretariat.interne@vlkh.net

03.-04.10.2019 Wien: Grundkurs Abdomenultraschall nach ÖGUM Leitung \& Auskunft: OA Dr. Ybinger, www.ultraschallkurse-wien.at
17.-19.10.2019 Wien: ÖGUM-Grundkurs Abdomenultraschall

Leitung \& Auskunft: Prof. Dr. Mostbeck, www.wienerultraschallseminare.at

06.11.2019 Baden bei Wien: Nephrologischer Ultraschallkurs

Leitung \& Auskunft: Prof. Dr. Gritzmann, www.sonoseminare.com 
07.11.2019 Baden bei Wien: UltraschallBasiskurs Abdomen

Leitung \& Auskunft: Prof. Dr. Gritzmann, www.sonoseminare.com

07.-09.11.2019 Hohenems: Abdomensonografie - Aufbau- und Abschlusskurs Leitung \& Auskunft: OA Dr. Gehmacher, sekretariat.interne@vlkh.net

22.-23.11.2019 Salzburg: ÖGUM-Grundkurs Abdomen mit Basisnotfallsonografie Leitung \& Auskunft: OA Dr. Hollerweger, kontakt@bhb-sonokurse.at

14.-15.12.2019 Wien: ÖGUM-Grundkurs Abdomensonografie

Leitung \& Auskunft: OA Dr. Aiginger, www.ultraschallkurs.at

\section{Bewegungsapparat}

09.-11.10.2019 Innsbruck: Musculoskeletal Sonography-Course for Rheumatologists - Basic Level

Leitung \& Auskunft: PD Dr. Dejaco, margit. bonatti@chello.at

12.10.2019 Amstetten: Ultraschallkurs Gelenke und Weichteile EFAST

Leitung \& Auskunft: Prof. Dr. Gritzmann, www.sonoseminare.com

\section{Echokardiografie}

19.09. 2019 Wien: Fokussierte Echokardiografie Leitung \& Auskunft: OA Dr. Aiginger, www.ultraschallkurs.at

10.-12.10.2019 Wien: Echokardiografie Grundkurs I + II inkl. Notfallsonografie mit praktischen Übungen

Leitung \& Auskunft: Dr. Huber,

www.boei.or.at

12.12.2019 Wien: Fokussierte Echokardiografie

Leitung \& Auskunft: OA Dr. Aiginger, www.ultraschallkurs.at

\section{Gefäße}

27.-28.09.2019 Salzburg: Karotissonografie Grundkurs Leitung \& Auskunft: Prim. Univ. Doz. Dr. Katzenschlager, www.boei.or.at
11.-12.10.2019 Salzburg: Karotissonografie Grundkurs

Leitung \& Auskunft: Prim. Univ. Doz. Dr.

Katzenschlager, www.boei.or.at

08.-09.11.2019 Baden bei Wien: Ultraschallkurs Halsgefäße und periphere

Gefäße

Leitung \& Auskunft: Prof. Dr. Gritzmann, www.sonoseminare.com

29.-30.11.2019 Innsbrucker Workshop für vaskuläre Sonografie

Leitung \& Auskunft: PD Dr. Gruber, PD Dr. Loizides, OA Dr. Sturm, Dr. Noeldeke, info@dis-innsbruck.com

13.12.2019 Wien: Grundkurs KarotisDuplex-Sonografie Leitung \& Auskunft: OA Dr. Aiginger, www.ultraschallkurs.at

\section{Gynäkologie}

15.-16.11.2019 Seggau: Pränatal-Symposium „Bunter Herbst in Seggau" Leitung \& Auskunft: Prof. Dr. Pertl, gyn-weststeiermark@kages.at

23.11.2019 Wien: Der geburtshilfliche Ultraschall - Tipps und Tricks für die Praxis mit hands-on-Übungen Leitung \& Auskunft: Dr. Burger, assam@arztnoe.at

\section{Kopf/Hals}

11.10.2019 Wien: Klinisch orientierte Schilddrüsensonografie einschließlich Schilddrüsenfunktionsstörung Leitung \& Auskunft: Prof. Dr. Blank, www.boei.or.at/kurse.htm, sekr@boei.or.at

\section{Nervensonografie}

22.-23.11.2019 Salzburg: 1. Internationaler ÖGUM/DEGUM-Nervenultraschallkurs, Sonografie des peripheren Nervensystems: "What Sonography can do for you!" Leitung \& Auskunft: Prof. Dr. A. Grimm, OA PD Dr. H. Gruber, PD Dr. S. Meng, OA PD Dr. A. Loizides, PD Dr. H. Platzgummer, www.oegumkurs.at

\section{Notfallsonografie}

07.09.2019 Graz: Notfallsonografie-Kurs/ EFAST-Kurs

Leitung \& Auskunft: Prof. Dr. Gritzmann, www.sonoseminare.com

19.-20.09.2019 Wien: ÖGUM-zertifizierte notfallsonografische Basisausbildung inklusive Megacode-Reanimationsübung Leitung \& Auskunft: OA Dr. Aiginger, www.ultraschallkurs.at

02.10.2019 Wien: Notfallsonografie EFAST Leitung \& Auskunft: OA Dr. Ybinger, www.ultraschallkurse-wien.at

03.-04.10.2019: Notfallsonografie und TTE in der Anästhesie und Intensivmedizin Leitung \& Auskunft: Dr. Gorsewski, www.perioptee-innsbruck.at\#

19.10.2019 Salzburg: NotfallsonografieKurs/EFAST-Kurs

Leitung \& Auskunft: Prof. Dr. Gritzmann, www.sonoseminare.com

11.12.2019 Wien: Notfallsonografie EFAST Leitung \& Auskunft: OA Dr. Aiginger, www.ultraschallkurs.at

\section{Pädiatrie/Bewegungsapparat}

11.-12.09.2019 Stolzalpe: Sonografie der Säuglingshüfte nach Graf Leitung \& Auskunft: PD Dr. Tschauner, OA Dr. Spieß, orthopaedie.sto@kages.at

04.-05.12.2019 Stolzalpe: Sonografie der Säuglingshüfte nach Graf Leitung \& Auskunft: PD Dr. Tschauner, OA Dr. Spieß, orthopaedie.sto@kages.at

\section{Interdisziplinär}

16.-19.10.2019 Leipzig/DE: Ultraschall 2019, 43. Dreiländertreffen der DEGUM/ ÖGUM/SGUM

Weitere Informationen unter www.ultraschall2019.de. 